\author{
Alessandro Caimi ${ }^{1}$ \\ Department of Electronics, \\ Information and Bioengineering, \\ Politecnico di Milano, \\ Milano 20133, Italy \\ e-mail: alessandro.caimi@polimi.it
}

\section{Francesco Sturla}

Department of Electronics, Information and Bioengineering

Politecnico di Milano,

Milano 20133, Italy

e-mail: francesco.sturla@polimi.it

Bryan Good

Department of Biomedical Engineering, The Pennsylvania State University,

State College, PA 16802

e-mail: bcg5069@psu.edu

\section{Marco Vidotto}

Department of Electronics, Information and Bioengineering,

Politecnico di Milano,

Milano 20133, Italy

e-mail: marco.vidotto@polimi.it

\section{Rachele De Ponti}

Department of Electronics, Information and Bioengineering,

Politecnico di Milano,

Milano 20133, Italy

e-mail: rachele.deponti@mail.polimi.it

Filippo Piatti

Department of Electronics, Information and Bioengineering,

Politecnico di Milano,

Milano 20133, Italy

e-mail: filippo.piatti@polimi.it

Keefe B. Manning

Mem. ASME

Department of Biomedical Engineering,

The Pennsylvania State University,

University Park, PA 16802

e-mail: kbm10@psu.edu

\author{
Alberto Redaelli \\ Mem. ASME \\ Department of Electronics, \\ Information and Bioengineering, \\ Politecnico di Milano, \\ Milano 20133, Italy \\ e-mail: alberto.redaelli@polimi.it
}

\section{Toward the Virtual} Benchmarking of Pneumatic Ventricular Assist Devices: Application of a Novel Fluid-Structure Interaction-Based Strategy to the Penn State 12 cc Device

The pediatric use of pneumatic ventricular assist devices (VADs) as a bridge to heart transplant still suffers for short-term major complications such as bleeding and thromboembolism. Although numerical techniques are increasingly exploited to support the process of device optimization, an effective virtual benchmark is still lacking. Focusing on the 12 cc Penn State pneumatic VAD, we developed a novel fluid-structure interaction (FSI) model able to capture the device functioning, reproducing the mechanical interplay between the diaphragm, the blood chamber, and the pneumatic actuation. The FSI model included the diaphragm mechanical response from uniaxial tensile tests, realistic VAD pressure operative conditions from a dedicated mock loop system, and the behavior of $V A D$ valves. Our FSI-based benchmark effectively captured the complexity of the diaphragm dynamics. During diastole, the initial slow diaphragm retraction in the air chamber was followed by a more rapid phase; asymmetries were noticed in the diaphragm configuration during its systolic inflation in the blood chamber. The FSI model also captured the major features of the device fluid dynamics. In particular, during diastole, a rotational wall washing pattern is promoted by the penetrating inlet jet with a lowvelocity region located in the center of the device. Our numerical analysis of the $12 \mathrm{cc}$ Penn State VAD points out the potential of the proposed FSI approach well resembling previous experimental evidences; if further tested and validated, it could be exploited as a virtual benchmark to deepen VAD-related complications and to support the ongoing optimization of pediatric devices. [DOI: 10.1115/1.4036936]

\section{Introduction}

Hypertrophic and dilated cardiomyopathies are the leading causes of heart failure (HF) [1]. Although less common than in

\footnotetext{
${ }^{1}$ Corresponding author.

Manuscript received January 27, 2017; final manuscript received May 18, 2017 published online June 16, 2017. Assoc. Editor: Ching-Long Lin.
}

adults, neonatal HF is associated with a higher level of mortality: $46 \%$ of infants affected by congenital defects, such as hypoplastic left heart syndrome, die within 5 years from birth [2].

To prevent neonatal HF, heart transplantation represents the optimal solution [3]. However, the availability of donor organs is not sufficient to meet the actual number of recipients on the waiting list, thus increasing the probability of death [4]. The use of VADs in pediatric patients has emerged as a valid bridge to heart 
transplant in the last decade [1], proving able to increase the survivor rate up to $90 \%$ [5]. However, the use of VADs for pediatric patients is feasible only in the short-term due to major complications, such as bleeding, infections, and thromboembolism [6,7].

In 2016, the first report of the Pediatric Interagency Registry for Mechanical Circulatory Support (PediMACS) analyzed survival outcomes and adverse event rates after durable VADs implantation in patients $(<19$ years of age, $n=200)$ enrolled between 2012 and 2015 [6]. Patients were treated with pulsatileflow devices $(n=91)$ and with continuous-flow ones $(n=109)$. Although at 6 months from VAD implant $87 \%$ of patients had a favorable outcome (58\% were transplanted), adverse events were reported with significant rates within 3 months: major bleeding $(n=59)$, device malfunctioning $(n=57)$, infection $(n=54)$, and neurologic events $(n=43)$. In particular, early adverse events were mostly predominant for pulsatile-flow devices, the majority of which were developed to be used in adults and adapted to patients-specific conditions. Indeed, the unique purposely designed pneumatic pediatric VAD (pVAD) that was adopted is the $10 \mathrm{cc}$ (body surface area, BSA $=0.45 \mathrm{~m}^{2}$ ) Berlin Heart EXCOR $^{\circledR}$ (Berlin Heart AG, Berlin, Germany) [7] since the available options for VAD support in children are still limited. Up to now, only the $10 \mathrm{cc}$ EXCOR $^{\circledR}$ VAD has received the Food and Drug Administration approval, but uniquely for a specific use (i.e., Investigational Design Exemption program).

In this context, following the success obtained from the development of the $70 \mathrm{cc}$ Pierce-Donachy VAD, which was sold worldwide by Thoratec (Thoratec, Pleasanton, CA) [8,9], the Pennsylvania State University began the development of a novel 12 cc Penn State pulsatile pVAD [10], specifically conceived for infants $\left(\mathrm{BSA}=0.5 \mathrm{~m}^{2}\right)$. This device, designed as a paracorporeal pneumatically driven system, consists of a blood compartment and an air chamber separated by a polymeric diaphragm. The alternate action of a vacuum pump and a compressor drives the pulsatile diaphragm motion, which in turn allows to fill and empty the blood compartment through two mechanical valves.

The ongoing VAD development and optimization relies on both in vitro [11-17] and in silico [18-25] approaches, which are nowadays exploited to deepen the device functioning and elucidate the determinants of reported VAD complications [6].

On the one hand, optical particle image velocimetry (PIV) is used as the gold standard to assess VAD fluid dynamics $[13,11,18]$ in terms of fluid velocity and near-wall shear rate. PIV can be combined with the high-speed video (HSV) technique to monitor the diaphragm kinematics under the actual operative conditions $[16,17]$. However, both the techniques require a dedicated in vitro setup [26] and a transparent VAD prototype. Acquired data are generally limited to planes parallel or normal to the diaphragm housing; shadow regions and optical complications, e.g., refraction and reflection drawbacks, may hamper the measurements.

On the other hand, in silico approaches have been proposed to investigate the pneumatic VAD functioning [20-22]. Marom et al. simplified the device pneumatic actuation adopting a user-defined law of motion [20]. However, as pointed out by Long et al. [22], the reliability of both diaphragm dynamics and device hemodynamics proved to depend on the reproduction of the device pneumatic actuation, which effect can be included through an effective fluid-structure interaction (FSI) approach. Besides capturing the strong coupling between the diaphragm and the two VAD chambers, three additional aspects are crucial to enhance the realism of the numerical FSI model: (i) the diaphragm mechanical response, (ii) the use of realistic operative working conditions of the device, and (iii) the modeling of VAD valves in the mitral and aortic ports.

First, the diaphragm mechanical response can be characterized performing uniaxial tensile tests on diaphragm specimens and can be reliably modeled as rate-independent, isotropic, and elastic $[19,27,28]$. Second, the actual operative working conditions can be collected testing the VAD in mock loop systems: subsequently, the numerical VAD model can be complemented using the extracted time-dependent waveforms of VAD operative pressures and blood flow rates as realistic boundary conditions [18,20,24].

Third, the realism of valves modeling can be achieved reproducing the geometry of the valves and their opening/closure behavior as a function of the surrounding flow velocity [20]. When the valves are omitted [22] or modeled as fixed in their fully open configuration [18,24], simplifications to include alternated ad hoc zero-velocity and resistance boundary conditions or a variable viscosity model are necessary $[18,25]$.

To the best of our knowledge, however, due to the complexity of the pneumatic actuation modeling and of the above-mentioned key points, none of the proposed numerical approaches accomplished to develop a comprehensive FSI model of pneumatic VADs.

In the present work, we sought to exploit a virtual FSI benchmark able to reproduce the complex pVAD functioning mechanism, so to quantitatively assess both the diaphragm dynamics and the fluid dynamics within the device. At this aim, we developed a novel FSI model of the Penn State $12 \mathrm{cc}$ pVAD combining the pneumatic actuation of the device, the diaphragm mechanical response, and in vitro pVAD operative working conditions.

\section{Methods}

The FSI model of the pVAD was implemented in the explicit solver LS-DYNA (Release 6.0, LSTC, Livermore, CA). The FSI algorithm, already adopted in the field of cardiovascular applications [29-34], is based on the "operator-split" approach available in LS-DYNA [35]. Herein, it was exploited to pinpoint the mechanical interplay occurring between the pVAD diaphragm and both the air and blood phases. Indeed, this method can couple nonconformal meshes for the fluid and solid domains, while the elements of the fluid are fixed and static. Therefore, this approach is not subjected to the remeshing and contact-related issues that affect arbitrary Lagrangian-Eulerian or cut-cell methods [33,36]. Similar to the immersed boundary method, the coupling algorithm transfers forces between the fluid and the solid grid, while no-slip conditions are indirectly imposed as in the fictitious domain method. The calculation of conservation equations is separated into a Lagrangian and an advection step. In the first Lagrangian step, the mesh can move with the flow, whereas in the second advection step, the mesh is remapped back to its original Eulerian position, and the flow between adjacent cells is computed by advection algorithm. The FSI coupling algorithm searches for penetrations between the fluid and structural meshes to apply a resistive force, linearly proportional to the penetrated distance, to both domains, thus preventing fluid leakage. Further details regarding the main features of the adopted FSI algorithm are available in Appendix A, within the electronic supplementary material, which is available under the "Supplemental Materials" tab for this paper on the ASME Digital Collection.

The FSI simulation was run on an Intel-Xeon $(2.93 \mathrm{GHz})$ workstation equipped with 12 processors with a computational expense of about $150 \mathrm{~h}$.

2.1 Device Geometrical Model and Discretization. Structural domain. The geometry of the pVAD model was extracted from the computer-aided design of the acrylic prototype (Fig. 1(a)) provided by the Artificial Heart Laboratory at The Penn State University (University Park, PA). The software GAMBIT (Ansys Inc., Canonsburg, PA) was used to reproduce both the pVAD diaphragm geometry and the inner-wall surface of the device, i.e., the pVAD case including the air and blood chambers (Figs. 1(b) and $1(c))$. The polymeric diaphragm was treated as a thin structure, prescribing a thickness equal to $0.4 \mathrm{~mm}$, as measured with a caliper. The pVAD model was then complemented by two Bjork-Shiley monostrut (BSM) tilting disk valves: Each BSM valve was modeled as a circular disk with a diameter of $12 \mathrm{~mm}$, a $15 \mathrm{deg}$ orientation angle, and an eccentric axis of rotation [14] (Fig. 1(d)). The diaphragm structural mesh was discretized, after 
(a)

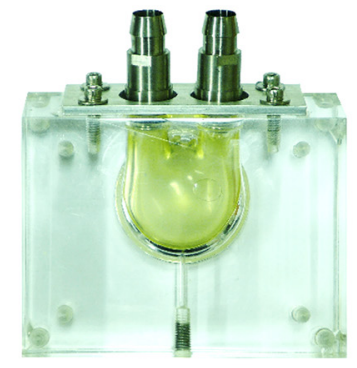

pVAD acrylic model (b)

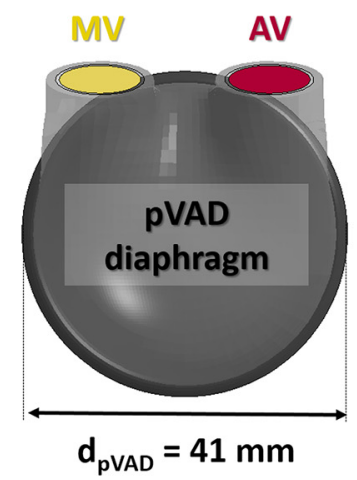

(c)

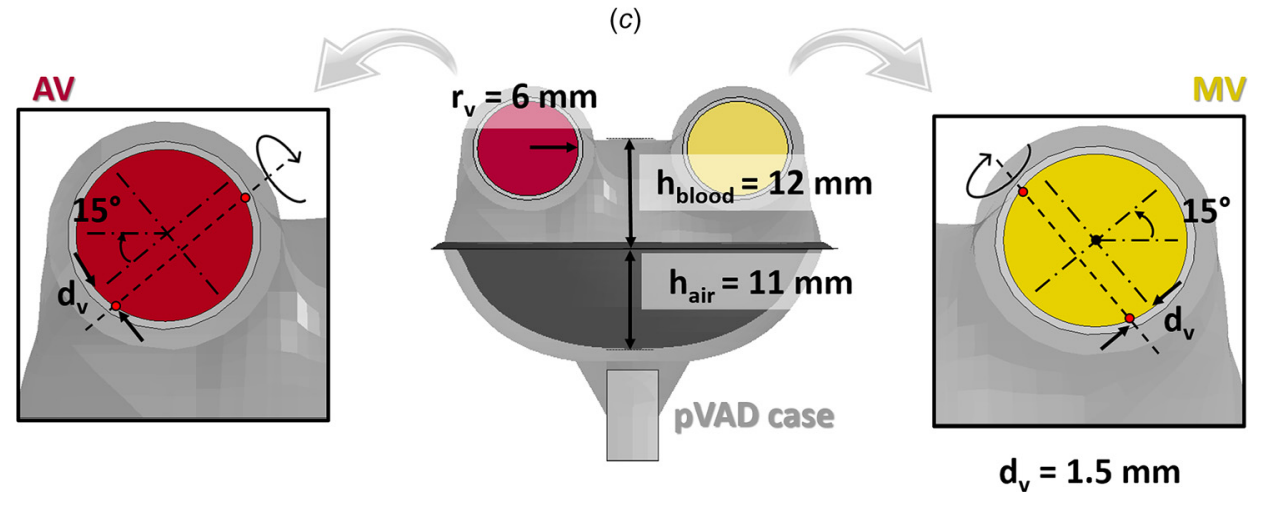

Fig. 1 Geometrical model of the Penn State 12 cc pVAD: (a) acrylic model of the device for in vitro tests at the Artificial Heart Lab; $(b)$ top view of the device pointing out the diaphragm diameter $\left(d_{\mathrm{pVAD}}\right)$ and the position of the mitral $(\mathrm{MV})$ and aortic (AV) ports; $(c)$ frontal view of the computer-aided design model reporting the height of both air $\left(h_{\text {air }}\right)$ and blood $\left(h_{\text {blood }}\right)$ chambers. A detail view of the tilting disk valves ( $r_{v}$, disk radius) is reported on the left (MV) and on the right (AV), highlighting the axis of rotation on each valve disk ( $d_{v}$ distance between the center of the valve and the rotational axis).

an acceptable level of grid convergence was obtained, into a structured mesh of 8731 quadrangular Belytschko-Tsay shell elements [37] and a characteristic dimension of $0.2-0.5 \mathrm{~mm}$ (for further details refer to Appendix B in the electronic supplementary material, which is available under the "Supplemental Materials" tab for this paper on the ASME Digital Collection). The pVAD case and the two BSM valves were discretized into 3270 and 1760 rigid shell elements, respectively.

Fluid domain. The fluid volume (Fig. 2(a)) was designed to embed the whole device, as requested by the FSI coupling algorithm. This control volume was subdivided into (i) the air compressor reservoir; (ii) the mitral and aortic blood reservoirs, positioned at the edge of the inlet and the outlet conduit, respectively. The entire fluid volume was discretized into a structured mesh of 73,710 hexahedral Eulerian elements with a single integration point [37] and a characteristic dimension of $0.4-0.8 \mathrm{~mm}$. This choice was based on a preliminary mesh-independence analysis performed on three fluid meshes: coarse (about 37k elements, characteristic dimension: $0.6-1.2 \mathrm{~mm}$ ), intermediate (i.e., the one adopted in the work), and fine (about 155k elements, characteristic dimension: $0.3-0.6 \mathrm{~mm}$ ). The comparison of the respective results in terms of blood velocity contour, pVAD diaphragm configuration, and mechanical stress showed no relevant differences in terms of field variables' distribution, and differences in peak values were within $3.5 \%$ between the intermediate and the fine mesh. In agreement with the criterion adopted in previous works [30,31], this level of mesh refinement was considered a reasonable tradeoff between the FSI computational expense and the need for reproducing physical phenomena involving large displacements and nonstationary flow field. Further details are available in Appendix B in the electronic supplementary material, which is available under the "Supplemental Materials" tab for this paper on the ASME Digital Collection.

2.2 Mechanical Properties. Diaphragm. The diaphragm within the pVAD is made of BioSpan ${ }^{\circledR}$ (DSM Inc., Berkeley, CA), i.e., a segmented polyether urethane urea. Uniaxial tensile tests were performed, according to the standard test methods (ASTM D638-01 and ASTM D1708-2a), on dog-bone specimens $(n=5)$ excised from the diaphragm. Experiments were carried out using a Lloyd tensile test machine (AMETEK TCI, Berwyn, PA) with a load cell of $5 \mathrm{~N}$ and a strain rate of $20 \mathrm{~mm} / \mathrm{min}$. The FSI simulation was performed assuming an isotropic linear elastic mechanical response for the diaphragm in order to reduce the computational cost of the analysis [31]. Although a maximum deformation lower than $10 \%$ was expected for the BioSpan ${ }^{\circledR}$ diaphragm [18], experimental data were fitted (Fig. 2(c)) in the range of $0-50 \%$ deformation, obtaining a Young's modulus $(E)$ equal to 7.0 MPa.

Fluids. Air was modeled as an ideal gas with a density of $1.29 \times 10^{-3} \mathrm{~kg} / \mathrm{m}^{3}$ while its thermodynamic energy function was controlled with the linear polynomial equation of state [38]. Blood was characterized as a Newtonian fluid with a density of $1060 \mathrm{~kg} / \mathrm{m}^{3}$ and a dynamic viscosity of $0.004 \mathrm{~Pa} \cdot \mathrm{s}$ while its thermodynamic energy function was controlled with the Gruneisen equation of state [32].

2.3 FSI Numerical Setup. The pVAD functioning was simulated throughout two consecutive systolic phases (340 ms each), including an intermediate diastolic cardiac phase $(460 \mathrm{~ms})$. At this purpose, realistic boundary conditions were measured under 

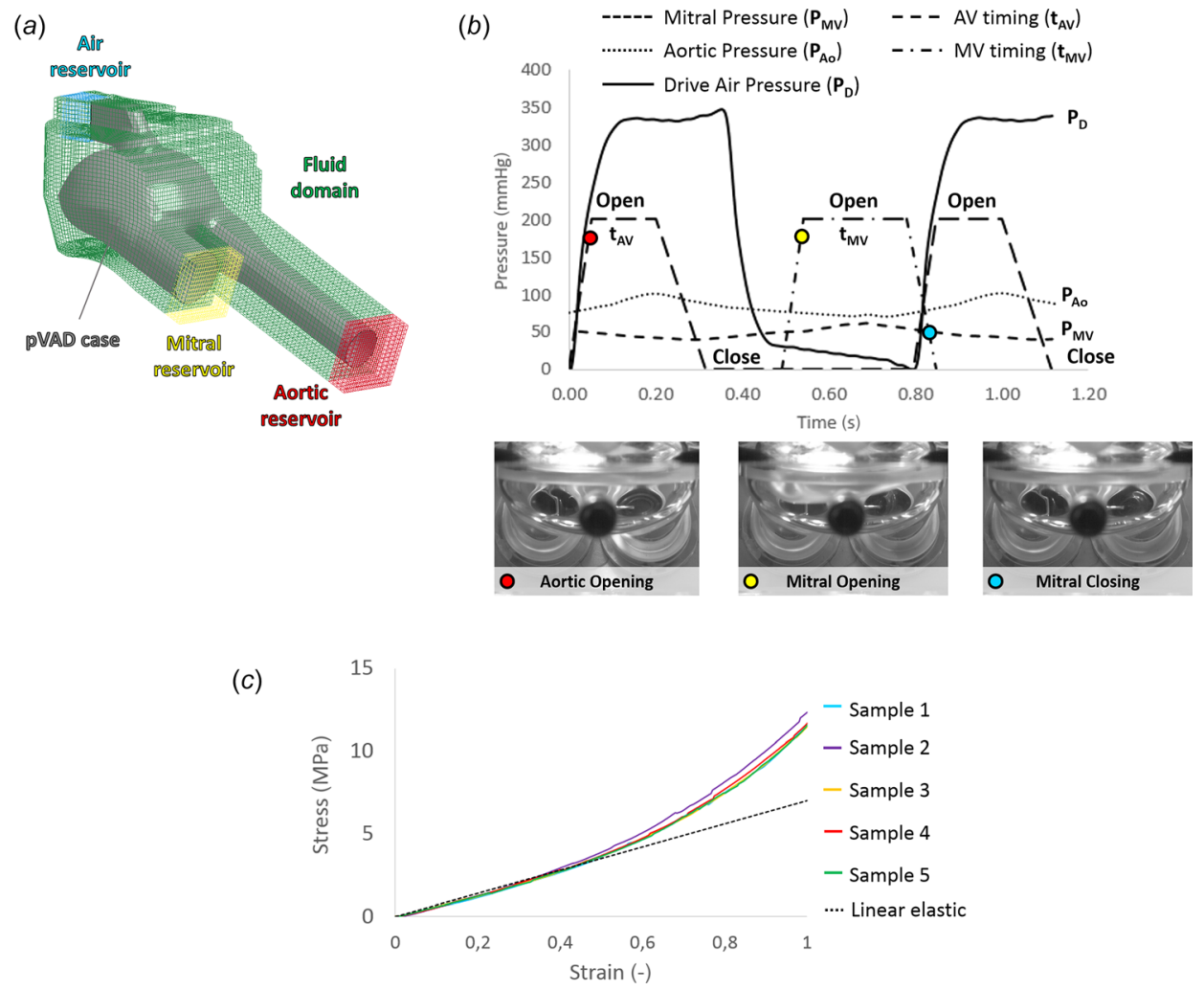

Fig. 2 (a) Numerical 12 cc pVAD FSI model: The pVAD case is immersed in a control volume consisting of the fluid domain (green grid), the air reservoir (light blue grid), the mitral (yellow grid), and the aortic (red grid) reservoirs. (b) Boundary conditions extracted from the in vitro benchmark and adopted in the FSI model: pressure time-dependent waveforms and the observed opening and closing timing of the tilting disk valves. (c) Experimental uniaxial tensile tests performed on the five diaphragm samples; (d) stress-strain response for each sample and the linear elastic approximation in the strain range $0-50 \%$ (see color figure online).

standard operative working conditions (heart rate: $75 \mathrm{bpm}$; cardiac output: $1.4 \mathrm{~L} / \mathrm{min}$ ) testing the acrylic pVAD in the experimental mock loop at the Artificial Heart Lab [26]. Pressure transducers (Maxxim Medical, Athens, TX) were used to extract the timedependent pressure waveforms of the air drive line $\left(P_{D}\right)$, aortic $\left(P_{\mathrm{Ao}}\right)$, and mitral $\left(P_{\mathrm{MV}}\right)$ reservoirs, respectively (Fig. 2(b)). Subsequently, these curves were applied as pressure loads in the FSI model: $P_{A o}$ and $P_{M V}$ were imposed to the fluid at the aortic reservoir (outlet) and mitral reservoir (inlet) of the fluid domain, respectively. $P_{D}$ was imposed at the air reservoir. Both opening and closure of the BSM valves timing were reproduced prescribing the time-dependent angular rotation of each tilting disk valve, as recorded on the experimental mock loop through HSV equipment (frame rate $=4 \mathrm{~ms}$ ) during $\mathrm{pVAD}$ normal functioning (Fig. 2(b)). The FSI coupling was modeled through the LS-DYNA penalty coupling algorithm based on the *CONSTRAINED_LAGRANGE_IN_SOLID keyword [37], as detailed in Appendix A.4 within the electronic supplementary material, which is available under the "Supplemental Materials" tab for this paper on the ASME Digital Collection.

\section{Results}

The FSI analysis of the pVAD functioning focused on the diaphragm dynamics and blood fluid dynamics within the device. Results were extracted from the simulated diastolic phase and the subsequent systolic phase.

3.1 Diaphragm Dynamics. Mechanical von Mises stress $\left(\sigma_{\mathrm{VM}}\right)$, circumferential $\left(\varepsilon_{\mathrm{circ}}\right)$, and radial strains $\left(\varepsilon_{\mathrm{rad}}\right)$ were computed from the diastolic filling phase $(650-800 \mathrm{~ms}$, Figs. $3(a)-3(d))$ to the systolic peak of blood ejection $(850-1000$ ms, Figs. 3(e)-3(h)). During diastole, $\sigma_{\mathrm{VM}}$ locally increased up to $2.2 \mathrm{MPa}$ on clearly visible folded regions of the diaphragm (Figs. 3(a)-3(c)) while at end-diastole $(800 \mathrm{~ms}$, Fig. 3(d)), the diaphragm was completely unfolded. During systole, due to the pneumatic actuation, the diaphragm progressively inflated into the blood chamber (Figs. 3(e)-3(g)): ring-shaped folded regions were visible with $\sigma_{\mathrm{VM}}$ reaching values proximal to $1.3 \mathrm{MPa}$. At peak systole (Fig. 3(h)), the diaphragm was fully stretched into the blood chamber and $\sigma_{\mathrm{VM}}$ stress significantly increased, in particular close to the aortic port, where a peak $\sigma_{\mathrm{VM}}$ value of about 2.4 MPa was noticed.

As regards diaphragm deformation, $\varepsilon_{\text {circ }}$ and $\varepsilon_{\text {rad }}$ ranged between $-5 \%$ and $+5 \%$ during diastole. During systole, the diaphragm stretching increased and $\varepsilon_{\text {circ }}$ reached a maximum value of $+10 \%$ at peak systole, close to the aortic port (Fig. 3(h)); in the same region, $\varepsilon_{\text {rad }}$ reported values largely below $-5 \%$ (Fig. $3(h)$, $t=1000 \mathrm{~ms})$.

Furthermore, we deepened the diaphragm motion comparing the time-dependent position $Y_{P}$ of the diaphragm nodes in the $y$-direction (i.e., normal to the plane of the diaphragm housing) along two diametrical and orthogonal axes (Fig. 4), i.e., parallel (red line) and perpendicular (blue line) to the valved pVAD side, respectively. At end-systole (Fig. 4, 550-1050 ms), the diaphragm showed an almost symmetrical and comparable shape on both the diametrical profiles, with a maximum distance of $2.4 \mathrm{~mm}$. On the contrary, the diametrical diaphragm profiles significantly differed and assumed an asymmetric configuration during the diastolic blood chamber filling $(t=750 \mathrm{~ms})$ and the systolic diaphragm inflation $(t=950 \mathrm{~ms})$ into the blood chamber. Computed 


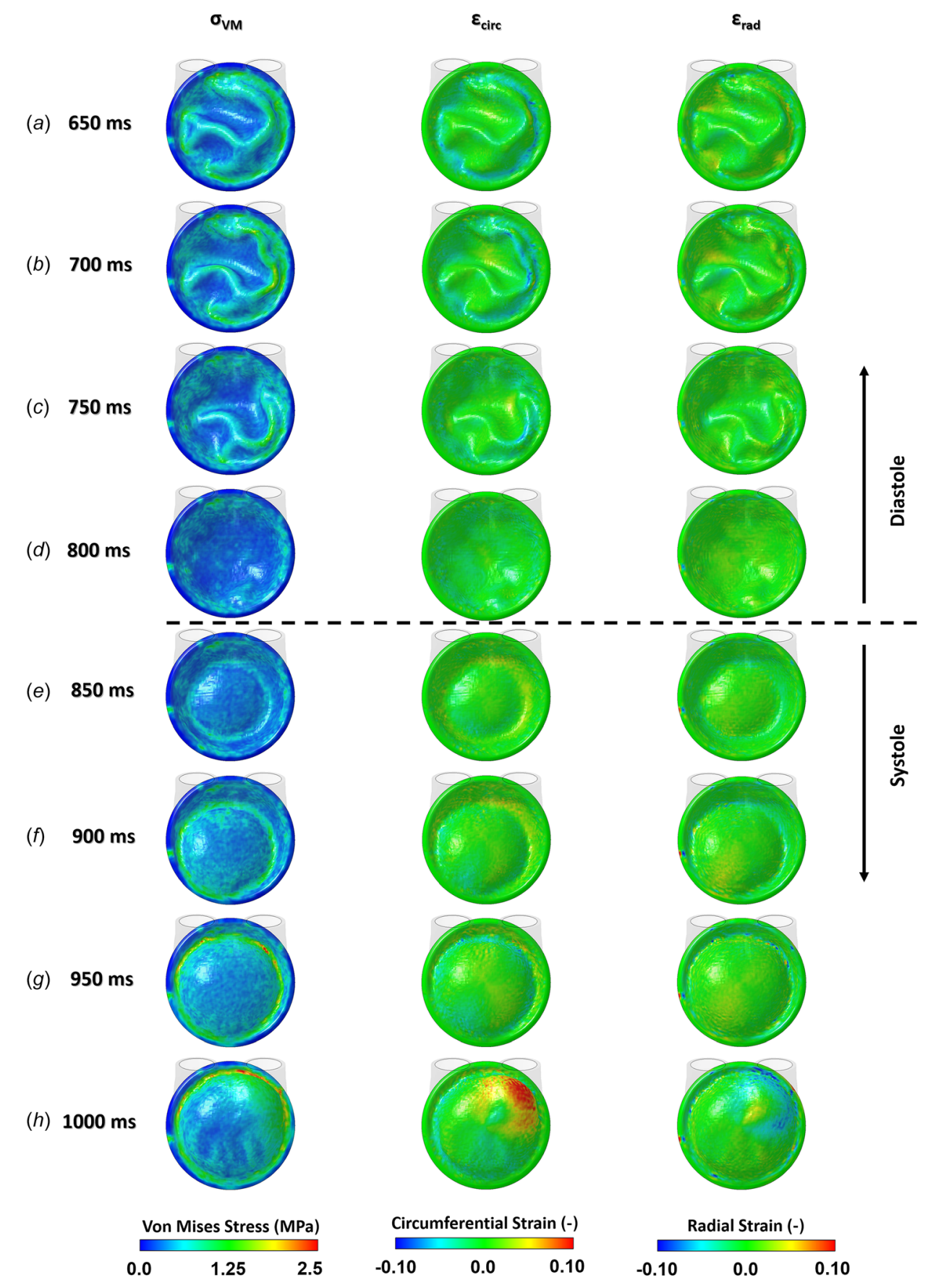

Fig. 3 Contour maps of von Mises stress $\left(\sigma_{\mathrm{VM}}\right.$, left column), circumferential strain $\left(\varepsilon_{\mathrm{circ}}\right.$, central column), and radial strain ( $\varepsilon_{\text {rad }}$, right column) computed on the pVAD diaphragm from diastolic pVAD filling ( $t=650 \mathrm{~ms})$ up to the peak of systolic ejection $(t=1000 \mathrm{~ms})$

discrepancies were equal to $4.1 \mathrm{~mm}$ at $750 \mathrm{~ms}$ and $5.8 \mathrm{~mm}$ at 950 $\mathrm{ms}$, respectively.

Also, we quantified the diaphragm opening $\left(D_{O}\right)$ during diastole $[16,17]$. Accordingly, we computed $Y_{P}$ for nine equidistant points, selected on the central portion of the diaphragm; during diastole, starting from the mitral port opening ( $t=500 \mathrm{~ms}$, early-diastole), each nodal displacement was normalized with respect to the corresponding value of displacement computed at the fully open diaphragm configuration $\left(t=800 \mathrm{~ms}\right.$, i.e., end-diastole). $D_{O}$ values are plotted in Fig. 4(b) as median, 10th and 90th percentiles: a slow filling phase was initially visible, with a $D_{O}$ equal to $5.3 \%$ after $100 \mathrm{~ms}$, while a rapid filling phase occurred in the remaining $150 \mathrm{~ms}$, with $D_{O}$ increasing up to $89.5 \%$.
3.2 pVAD Fluid Dynamics. The FSI simulation showed a good agreement with the experimental flow rate waveforms (Fig. 5(a)) measured on the mock loop system for both the mitral inflow and the aortic outflow. At the inflow, the maximum numerical diastolic flow rate was equal to $5.63 \mathrm{~L} / \mathrm{min}$, which is $9.1 \%$ higher than the experimental value of $5.16 \mathrm{~L} / \mathrm{min}$. At the aortic outflow, we computed a maximum systolic numerical flow rate of $5.92 \mathrm{~L} / \mathrm{min}$, underestimating the corresponding in vitro value equal to $5.96 \mathrm{~L} / \mathrm{min}$ by $0.7 \%$. Although resembling the timing and the peak values of the experimental flow rates, the negative flow rate preceding the complete BMS valves closure was not captured.

In Fig. 5, blood velocity contour maps are reported on a cut-section view, parallel to the diaphragm housing plane and 


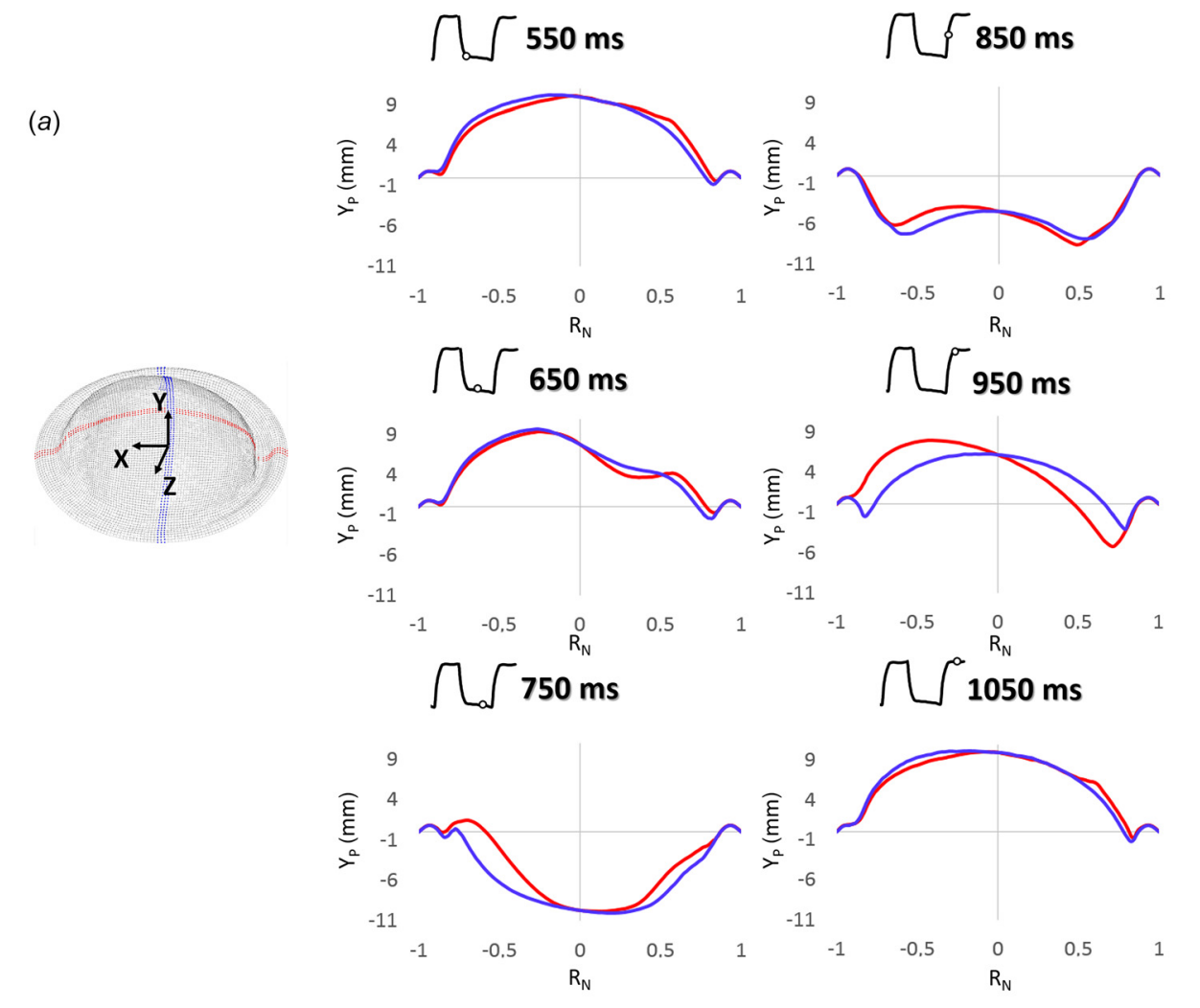

(b)

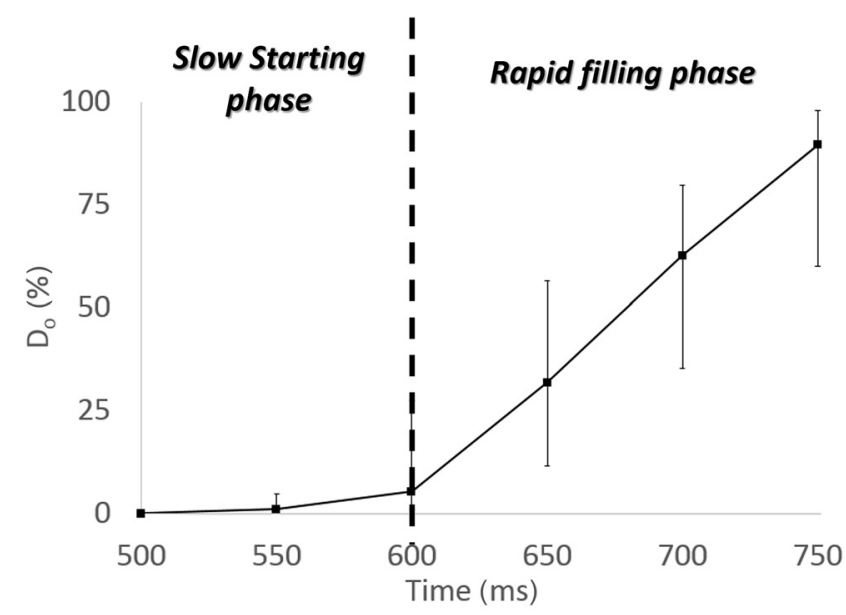

Fig. 4 (a) Time course of the diaphragm profile, along two diametrical principal axes, during both diastole (550-750 ms) and systole (850-1050 ms). For each point of the profile, the $Y_{p}$ coordinate, i.e., normal to the diaphragm housing plane, and $\boldsymbol{R}_{\boldsymbol{N}}$, i.e., normalized membrane radius, are computed. $(b)$ Diastolic diaphragm opening $\left(D_{O}\right)$ averaged on nine equidistant points selected on the central portion of the diaphragm (see color figure online).

positioned to a distance of $11 \mathrm{~mm}$ from the VAD ports. During diastole (650-800 ms), a double inlet jet entered the blood chamber through the mitral BSM valve and reached a velocity peak value of $1.4 \mathrm{~m} / \mathrm{s}$ (Fig. 5(b), $t=650 \mathrm{~ms}$ ). Subsequently, due to diaphragm retraction in the air chamber, the high-velocity jet propagated within the blood chamber following a circular pattern, i.e., parallel to the wall of the chamber, with a peak of velocity ranging between 0.6 and $0.8 \mathrm{~m} / \mathrm{s}$ (Fig. 5(b), 700-750 ms). At end-diastole (Fig. 5(b), $t=800 \mathrm{~ms}$ ), the circular jet reached the aortic port and its velocity decreased below $0.5 \mathrm{~m} / \mathrm{s}$ while a low-velocity region $(0.1-0.2 \mathrm{~m} / \mathrm{s})$, it was visible in the center of the chamber, thus reproducing the typical diastolic washing pattern of the device [39]. Finally, during systolic ejection (Figs. 5(g) and 5(h)), the diaphragm was inflated in the blood chamber and blood rapidly flowed through the aortic valve, reaching a peak velocity of $1.4 \mathrm{~m} / \mathrm{s}$. In particular, we focused on three different diastolic frames $(600 \mathrm{~ms}, 700 \mathrm{~ms}$, and $800 \mathrm{~ms})$ to compute (i) the pathlines of neutrally buoyant particles seeded from the mitral port (Figs. 6(a)-6(c)); (ii) the isosurfaces of blood velocity magnitude at $0.5 \mathrm{~m} / \mathrm{s}$ and $1.0 \mathrm{~m} / \mathrm{s}$ (Figs. $6(d)-6(f)$ ). The blood initially filled a small portion of the blood chamber since the diaphragm is still inflated in the blood chamber $(t=600 \mathrm{~ms})$. Then, the rapid retraction of the diaphragm in the air case increased the diastolic filling and blood rapidly moved along the chamber wall (Figs. 6(b) and $6(c)$ ) between $700 \mathrm{~ms}$ and $800 \mathrm{~ms}$. This circular pattern washed the wall of the blood chamber along its entire extension (Fig. 6(f), $t=800 \mathrm{~ms}$ ) as depicted by the $0.5 \mathrm{~m} / \mathrm{s}$ velocity isosurface. 
(a)

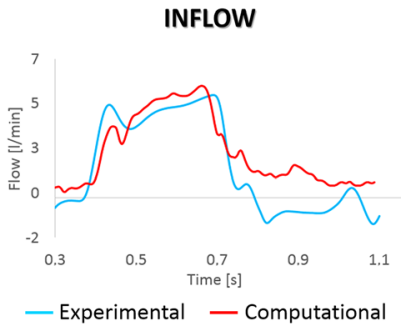

OUTFLOW

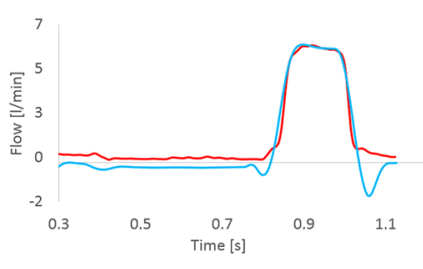

(b)

I
I
I
I
I
I
I
I
I
I
I
I
I
I
I
I
I
I
I
I
I
I
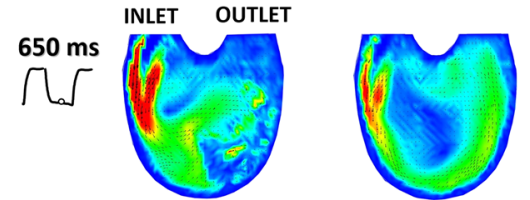

$800 \mathrm{~ms}$

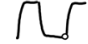

$700 \mathrm{~ms}$
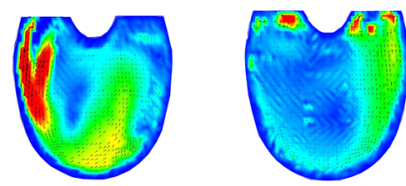

$850 \mathrm{~ms}$

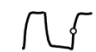

$750 \mathrm{~ms}$
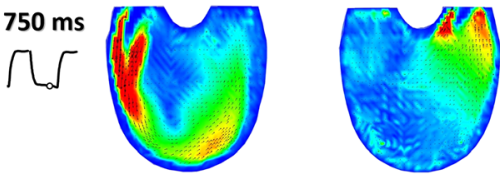

$900 \mathrm{~ms}$

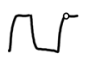

Fig. 5 (a) Time-dependent waveforms of flow rate extracted at the inflow, i.e., mitral valve (top panel), and at the outflow, i.e., aortic valve (bottom panel), from the in vitro mock loop (light blue) and from the numerical model (red); (b) contour maps of the velocity field computed at the $11 \mathrm{~mm}$ parallel plane cross section during the diastolic filling phase of the device $(t=650,700,750,800 \mathrm{~ms})$ and the first instants of the systolic ejection phase $(t=850,900 \mathrm{~ms})$ (see color figure online)

\section{Discussion}

In the present work, we assessed the functioning of the $12 \mathrm{cc}$ Penn State pneumatic VAD through a novel FSI-based approach able to reproduce the strong coupling between the diaphragm, the blood chamber, and the pneumatic actuation. In particular, we combined the FSI coupling approach with in vitro ground-truth data in order to define a virtual benchmark for the numerical assessment of the device. Indeed, we complemented the FSI model exploiting our in vitro facilities $[12,17,26]$ : realistic air and blood pressure operative conditions were collected on the VAD prototype, mounted on a dedicated mock loop system and equipped with pressure transducers; the opening and closure behavior of VAD valves were estimated using HSV acquisitions; finally, samples of the BioSpan ${ }^{\circledR}$ material were employed for uniaxial tensile tests to quantify the diaphragm mechanical response.

To the best of our knowledge, this is the first attempt to improve the realism of numerical VAD modeling by means of an FSI-based approach able to capture the complexity of the device. Although a systematic in vitro validation of the FSI simulation was beyond the aims of the study, the FSI results were preliminary compared with in vitro data from the Artificial Heart Laboratory at The Penn State University (University Park, PA) [14-17], as reported in Fig. 7.

The systolic inflated configuration of the diaphragm well resembled the experimental one in terms of maximum diaphragm excursion (i.e., maximum $Y_{P}$ coordinate), as measured from HSV data on both the diametrical diaphragm axes (Fig. 7(a)). Numerical $Y_{P}$ results were equal to 9.64 and $10.12 \mathrm{~mm}$, underestimating of $5.5 \%$ and $5.4 \%$ the corresponding HSV values (10.20 and $10.70 \mathrm{~mm}$ ). The diaphragm asymmetrical inflation (Fig. 4(a)) was paralleled by a more pronounced stretch of the diaphragm toward the aortic port (Fig. 3(h)). Also, we quantified the diaphragm opening $\left(D_{O}\right.$, Fig. $\left.4(b)\right)$ during VAD diastolic filling, and we highlighted the presence of a starting slow phase followed by a more rapid filling [17]. Of note, the variability of the $D_{O}$ measure (between 10th and 90th percentiles) confirms the asymmetric configuration assumed by the diaphragm.
Such an accurate description of the diaphragm motion was reported to be crucial to reproduce physiological flow conditions $[16,20]$. As a result, the computed pattern and magnitude of the blood velocity field within the device (Fig. 5(b)) resemble previous evidences of conventional bidimensional quantitative PIV mapping $[11,13,15,17]$.

Specifically, our FSI model reproduced the concentration of the diastolic inlet jet through the major orifice of the BSM mitral valve [15], i.e., the orifice nearest to the outer perimeter of the device. For instance, as compared with PIV data (Fig. 7(b)), the FSI model captured the pattern and the entity of the velocity field resulting from the diastolic jet blood penetration, with a peak velocity equal to $0.86 \mathrm{~m} / \mathrm{s}$, overestimating of $7 \%$ the corresponding PIV data. Accordingly, the penetrating inlet jet promoted the formation of a rotational flow within the body of the device, qualitatively comparable to the one visible in PIV data (Fig. 7(b), left panel): this mid-to-late diastole rotational washing flow is recognized as a major feature of the pulsatile device [40]. Also, the FSI model exhibited a low-velocity region located in the center of the device (Fig. 5(b), $t=750-800 \mathrm{~ms}$ ): although more extended with respect to PIV data (Fig. 7(b), left panel), this low-velocity region was characterized by a comparable range of velocity below $0.2 \mathrm{~m} / \mathrm{s}$. During systole, both FSI results and PIV reported a highvelocity region proximal to the aortic port, while progressively decreasing moving from the aortic side toward the mitral side of the device (Fig. 7(b), right panel).

Hence, taking advantage of the virtual FSI benchmark, we adopted the $12 \mathrm{cc}$ Penn State VAD; three main practical implications can be suggested.

First, the FSI model can provide the time-dependent stress-strain response of the diaphragm (Fig. 3) and assess the VAD fluid dynamics as a direct result of the diaphragm motion. The former can be exploited to test the mechanical performance of novel surface-modified segmented polyether urethane urea materials, specifically developed to improve the diaphragm thromboresistance [41]. The latter, e.g., through the computation of 3D velocity pathlines (Figs. 6(a)-6(c)) and isosurfaces (Figs. 

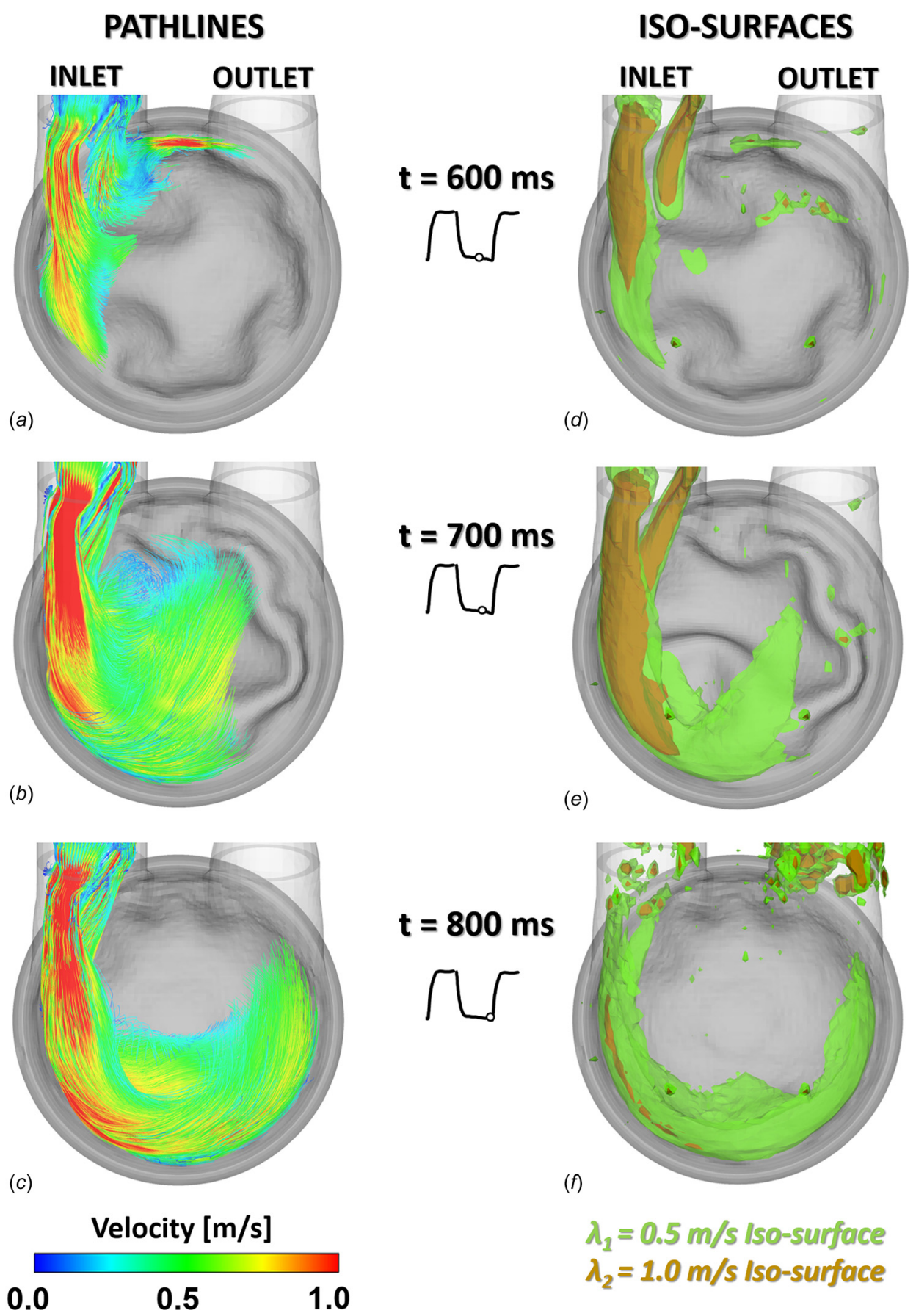

$$
\begin{aligned}
& \lambda_{1}=0.5 \mathrm{~m} / \mathrm{s} \text { Iso-surface } \\
& \lambda_{2}=1.0 \mathrm{~m} / \mathrm{s} \text { Iso-surface }
\end{aligned}
$$

Fig. 6 Visualization of the 3D fluid dynamics of the device during the diastolic filling phase $(t=600,700,800 \mathrm{~ms})$ by means of $(a)$ pathlines injected from the location of the mitral port and $(b)$ two velocity magnitude isosurfaces $\left(\lambda_{1}=0.5 \mathrm{~m} / \mathrm{s}\right.$, brown; $\lambda_{2}=1 \mathrm{~m} / \mathrm{s}$, green) (see color figure online)

6(d)-6(f)), can effectively pinpoint the high 3D flow field originating in the device and the peculiar rotational wall washing pattern of the device [40]. A visualization of the computed 3D pathlines originating within the $12 \mathrm{cc}$ Penn State pVAD is available online, in Appendix C within the electronic supplementary material, which is available under the "Supplemental Materials" tab for this paper on the ASME Digital Collection.

Second, a realistic FSI strategy could allow a systematic VAD optimization and may precede in vitro tests on mock loop systems, also limiting the necessary prototyping cost [40]. As a matter of fact, the simple rescaling of adult VADs is not sufficient to develop an effective pediatric device: in the downscaled version of the 70 cc Penn State VAD [42], the internal fluid dynamics was significantly altered with respect to the regular $70 \mathrm{cc}$ device, and a high degree of thrombus formation was reported. At this aim, appropriate adjustment of the VAD design can significantly improve the device performance [21], suggesting that an effective in silico VAD benchmarking could facilitate and support the subsequent in vitro and in vivo animal evaluation of the device $[10,12,43]$.

Third, the proposed FSI approach can be useful to support computational fluid dynamics (CFD) model predictions. Indeed, the moving flexible diaphragm, driving the pneumatic VAD blood flow, is the most important but also the most difficult VAD component to simulate [44]. Overcoming reported simplifications $[24,45]$ of the problem, we can benefit from the outcomes of our 
(a)
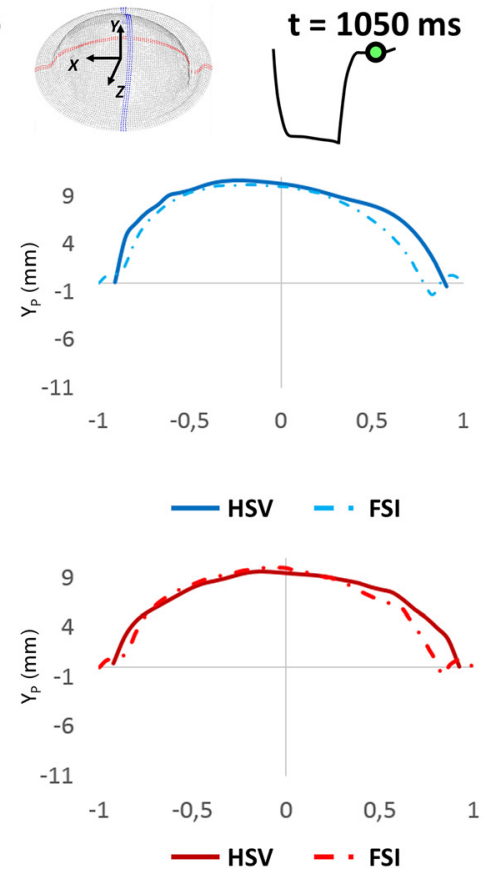

(b)

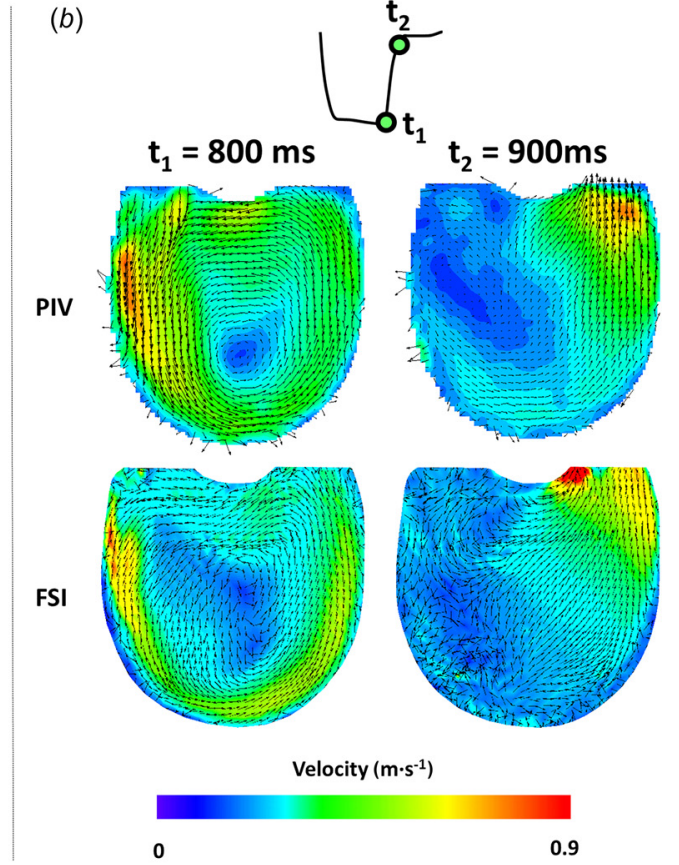

Fig. 7 Preliminary comparison between the computed FSI results and ground-truth in vitro data collected on the $12 \mathrm{cc}$ Penn State prototype: (a) maximum diaphragm excursion (i.e., $Y_{P}$ coordinate) at systole, as computed on both the diametrical diaphragm axes from the FSI model (dashed line) and high-speed video acquisitions (continuous line); (b) fluid dynamic comparison between the simulated FSI model and data from particle image velocimetry, during diastole (left panel) and systole (right panel), respectively

FSI model and impose the realistic diaphragm kinematics as a boundary "wall motion" condition in a CFD model. Then, the invaluable CFD capabilities and accuracy can be exploited to pinpoint details of the flow field, such as regions of recirculation or stagnation, and to investigate, through inclusion of blood damage models, the exposure of red blood cells and platelets to fluid dynamic forces, which promote hemolysis as well as platelet activation and clotting $[44,46]$.

Two main limitations of our FSI modeling approach should be taken into consideration when interpreting results.

First, although the proposed FSI model resembled the main features (e.g., timing and peak value) of the experimental flow rates at the mitral and aortic ports of the device, the negative flow rate preceding the complete BMS valves closure was not captured. In our previous work [31], such a level of approximation in reproducing the exact experimental flow rate did not prevent the model from obtaining an acceptable level of agreement with experimental ground-truth data. We believe that two main issues need to be tackled in the future to reproduce the negative blood flow rates: (i) a more detailed numerical modeling of BMS valves, including the exact geometry of the BMS oscillating disk and the surrounding housing system [20]; (ii) the replacement of the BMS user-defined opening and closure behavior [20,22] with a fluid-driven passive actuation based on the FSI coupling algorithm.

Second, the adopted FSI approach required to discretize the Eulerian fluid domain with a mapped and uniformly distributed mesh, which was coarser than similar CFD studies [24,47], and it could not be refined near the walls and moving structures. Therefore, the flow gradients, e.g., occurring along the wall of the blood chamber, may not be accurately captured as like within highly refined boundary layers' regions in a CFD analysis. However, our FSI model captured the major features of the blood chamber bulk flow and the diaphragm dynamics with a good level of approximation.

In conclusion, the results of the analysis on the $12 \mathrm{cc}$ Penn State VAD underline the potential of the proposed FSI approach, although FSI modeling should be still considered an approximated method with respect to CFD modeling, which accuracy is beyond dispute. Nonetheless, if further tested and validated, the proposed FSI method could be of use to support the ongoing optimization of pediatric devices, as an additional virtual benchmark per se or as a valuable "bridge" to focused CFD analysis.

\section{Acknowledgment}

This study was partially supported by the U.S. National Institutes of Health through Grant No. HL108123.

\section{Nomenclature}

$\mathrm{BSA}=$ body surface area

$\mathrm{BSM}=$ Bjork-Shiley monostrut

$\mathrm{CFD}=$ computational fluid dynamics

$D_{o}=$ diaphragm opening

$E=$ Young's modulus

$\mathrm{FSI}=$ fluid-structure interaction

$\mathrm{HF}=$ heart failure

$\mathrm{HSV}=$ high-speed video

$P_{\text {Ao }}=$ aortic pressure

$P_{D}=$ driveline pressure

$P_{\mathrm{MV}}=$ mitral valve pressure

$\mathrm{pVAD}=$ pediatric ventricular assist device

PediMACS $=$ Pediatric Interagency Registry for Mechanical Circulatory Support

PIV $=$ particle image velocimetry

$\mathrm{VAD}=$ ventricular assist device

$3 \mathrm{D}=$ three-dimensional

$\varepsilon_{\text {circ }}=$ circumferential strain

$\varepsilon_{\text {rad }}=$ radial strain

$\sigma_{\mathrm{VM}}=$ Von Mises stress

\section{References}

[1] Colvin, M., Smith, J. M., Skeans, M. A., Edwards, L. B., Uccellini, K., Snyder, J. J., Israni, A. K., and Kasiske, B. L., 2017, "OPTN/SRTR 2015 Annual Data Report: Heart,” Am. J. Transplant., 17(Suppl. 1), pp. 286-356. 
[2] Fraser, C. D., Jr., Jaquiss, R. D., Rosenthal, D. N., Humpl, T., Canter, C. E., Blackstone, E. H., Naftel, D. C., Ichord, R. N., Bomgaars, L., Tweddell, J. S., Massicotte, M. P., Turrentine, M. W., Cohen, G. A., Devaney, E. J., Pearce, F. B., Carberry, K. E., Kroslowitz, R., and Almond, C. S., 2012, "Prospective Trial of a Pediatric Ventricular Assist Device," N. Engl. J. Med., 367(6), pp. 532-541.

[3] Canter, C. E., Shaddy, R. E., Bernstein, D., Hsu, D. T., Chrisant, M. R., Kirklin, J. K., Kanter, K. R., Higgins, R. S., Blume, E. D., Rosenthal, D. N., Boucek, M. M., Uzark, K. C., Friedman, A. H., and Young, J. K., 2007, "Indications for Heart Transplantation in Pediatric Heart Disease: A Scientific Statement From the American Heart Association Council on Cardiovascular Disease in the Young; the Councils on Clinical Cardiology, Cardiovascular Nursing, and Cardiovascular Surgery and Anesthesia; and the Quality of Care and Outcomes Research Interdisciplinary Working Group," Circulation, 115(5), pp. 658-676.

[4] Mah, D., Singh, T. P., Thiagarajan, R. R., Gauvreau, K., Piercey, G. E., Blume, E. D., Fynn-Thompson, F., and Almond, C. S., 2009, "Incidence and Risk Factors for Mortality in Infants Awaiting Heart Transplantation in the USA," J. Heart Lung Transplant., 28(12), pp. 1292-1298.

[5] Potapov, E. V., Stiller, B., and Hetzer, R., 2007, "Ventricular Assist Devices in Children: Current Achievements and Future Perspectives," Pediatr. Transplant., 11(3), pp. 241-255.

[6] Blume, E. D., Rosenthal, D. N., Rossano, J. W., Baldwin, J. T., Eghtesady, P., Morales, D. L., Cantor, R. S., Conway, J., Lorts, A., Almond, C. S., Naftel, D. C., and Kirklin, J. K., 2016, "Outcomes of Children Implanted With Ventricular Assist Devices in the United States: First Analysis of the Pediatric Interagency Registry for Mechanical Circulatory Support (PediMACS)," J. Heart Lung Transplant., 35(5), pp. 578-584.

[7] Morales, D. L., Almond, C. S., Jaquiss, R. D., Rosenthal, D. N., Naftel, D. C. Massicotte, M. P., Humpl, T., Turrentine, M. W., Tweddell, J. S., Cohen, G. A., Kroslowitz, R., Devaney, E. J., Canter, C. E., Fynn-Thompson, F., Reinhartz, O., Imamura, M., Ghanayem, N. S., Buchholz, H., Furness, S., Mazor, R., Gandhi, S. K., and Fraser, C. D., Jr., 2011, "Bridging Children of All Sizes to Cardiac Transplantation: The Initial Multicenter North American Experience With the Berlin Heart EXCOR Ventricular Assist Device," J. Heart Lung Transplant., 30(1), pp. 1-8.

[8] Gaines, W. E., Pierce, W. S., Donachy, J. H., Rosenberg, G., Landis, D. L. Richenbacher, W. E., and Waldhausen, J. A., 1985, "The Pennsylvania State University Paracorporeal Ventricular Assist Pump: Optimal Methods of Use," World J. Surg., 9(1), pp. 47-53.

[9] McBride, L. R., Naunheim, K. S., Fiore, A. C., Moroney, D. A., and Swartz, M T., 1999, "Clinical Experience With 111 Thoratec Ventricular Assist Devices," Ann. Thorac. Surg., 67(5), pp. 1233-1238.

[10] Weiss, W. J., Carney, E. L., Clark, J. B., Peterson, R., Cooper, T. K., Nifong, T. P., Siedlecki, C. A., Hicks, D., Doxtater, B., Lukic, B., Yeager, E., Reibson, J., Cysyk, J., Rosenberg, G., and Pierce, W. S., 2012, "Chronic In Vivo Testing of the Penn State Infant Ventricular Assist Device," ASAIO J., 58(1), pp. $65-72$.

[11] Cooper, B. T., Roszelle, B. N., Long, T. C., Deutsch, S., and Manning, K. B. 2010, "The Influence of Operational Protocol on the Fluid Dynamics in the 12 cc Penn State Pulsatile Pediatric Ventricular Assist Device: The Effect of EndDiastolic Delay," Artif. Organs, 34(4), pp. E122-E133.

[12] Deutsch, S., Tarbell, J. M., Manning, K. B., Rosenberg, G., and Fontaine, A. A., 2006, "Experimental Fluid Mechanics of Pulsatile Artificial Blood Pumps," Annu. Rev. Fluid Mech., 38(1), pp. 65-86.

[13] Roszelle, B. N., Cooper, B. T., Long, T. C., Deutsch, S., and Manning, K. B., 2008, "The 12 cc Penn State Pulsatile Pediatric Ventricular Assist Device: Flow Field Observations at a Reduced Beat Rate With Application to Weaning," ASAIO J., 54(3), pp. 325-331.

[14] Roszelle, B. N., Deutsch, S., and Manning, K. B., 2010, "A Parametric Study of Valve Orientation on the Flow Patterns of the Penn State Pulsatile Pediatric Ventricular Assist Device," ASAIO J., 56(4), pp. 356-363.

[15] Roszelle, B. N., Deutsch, S., and Manning, K. B., 2010, "Flow Visualization of Three-Dimensionality Inside the $12 \mathrm{cc}$ Penn State Pulsatile Pediatric Ventricular Assist Device," Ann. Biomed. Eng., 38(2), pp. 439-455.

[16] Hochareon, P., Manning, K. B., Fontaine, A. A., Deutsch, S., and Tarbell, J. M., 2003 "Diaphragm Motion Affects Flow Patterns in an Artificial Heart," Artif. Organs, 27(12), pp. 1102-1109.

[17] Schonberger, M., Deutsch, S., and Manning, K. B., 2012, "The Influence of Device Position on the Flow Within the Penn State $12 \mathrm{cc}$ Pediatric Ventricular Assist Device," ASAIO J., 58(5), pp. 481-493.

[18] Avrahami, I., Rosenfeld, M., Raz, S., and Einav, S., 2006, "Numerical Model of Flow in a Sac-Type Ventricular Assist Device," Artif. Organs, 30(7), pp. 529-538.

[19] Donahue, T. L., Rosenberg, G., Jacobs, C. R., and Weiss, W. J., 2003, "Finite Element Analysis of Stresses Developed in Blood Sacs of a Pusherplate Blood Pump," Comput. Methods Biomech. Biomed. Eng., 6(1), pp. 7-15.

[20] Marom, G., Chiu, W. C. Crosby, J. R., DeCook, K. J., Prabhakar, S., Horner, M., Slepian, M. J., and Bluestein, D., 2014, "Numerical Model of Full-Cardiac Cycle Hemodynamics in a Total Artificial Heart and the Effect of Its Size on Platelet Activation," J. Cardiovasc. Transl. Res., 7(9), pp. 788-796.

[21] Long, C. C., Marsden, A. L., and Bazilevs, Y., 2014, "Shape Optimization of Pulsatile Ventricular Assist Devices Using FSI to Minimize Thrombotic Risk," Comput. Mech., 54(4), pp. 921-932.
[22] Long, C. C., Marsden, A. L., and Bazilevs, Y., 2013, "Fluid-Structure Interaction Simulation of Pulsatile Ventricular Assist Devices," Comput. Mech., 52(5), pp. $971-981$

[23] Haut Donahue, T. L., Dehlin, W., Gillespie, J., Weiss, W. J., and Rosenberg, G., 2009, "Finite Element Analysis of Stresses Developed in the Blood Sac of a Left Ventricular Assist Device,” Med. Eng. Phys., 31(4), pp. 454-460.

[24] Medvitz, R. B., Kreider, J. W., Manning, K. B., Fontaine, A. A., Deutsch, S. and Paterson, E. G., 2007, "Development and Validation of a Computationa Fluid Dynamics Methodology for Simulation of Pulsatile Left Ventricular Assist Devices," ASAIO J., 53(2), pp. 122-131.

[25] Avrahami, I., Rosenfeld, M., and Einav, S., 2006, "The Hemodynamics of the Berlin Pulsatile VAD and the Role of Its MHV Configuration," Ann. Biomed. Eng., 34(9), pp. 1373-1388.

[26] Rosenberg, G., Phillips, W., Landis, D., and Pierce, W. S., 1981, "Design and Evaluation of the Pennsylvania State University Mock Circulatory System," ASAIO J., 4(2), pp. 41-49.

[27] Abraham, G. A., Frontini, P. M., and Cuadrado, T. R., 1997, "Physical and Mechanical Behavior of Sterilized Biomedical Segmented Polyurethanes,' J. Appl. Polym. Sci., 65(6), pp. 1193-1203.

[28] Roland, C. M., Twigg, J. N., Vu, Y., and Mott, P. H., 2007, "High Strain Rate Mechanical Behavior of Polyurea," Polymer, 48(2), pp. 574-578.

[29] Wu, W., Pott, D., Mazza, B., Sironi, T., Dordoni, E., Chiastra, C., Petrini, L., Pennati, G., Dubini, G., Steinseifer, U., Sonntag, S., Kuetting, M., and Migliavacca, F., 2016, "Fluid-Structure Interaction Model of a Percutaneous Aortic Valve: Comparison With an In Vitro Test and Feasibility Study in a PatientSpecific Case," Ann. Biomed. Eng., 44(2), pp. 590-603.

[30] Sturla, F., Votta, E., Stevanella, M., Conti, C. A., and Redaelli, A., 2013 , "Impact of Modeling Fluid-Structure Interaction in the Computational Analysis of Aortic Root Biomechanics," Med. Eng. Phys., 35(12), pp. 1721-1730.

[31] Piatti, F., Sturla, F., Marom, G., Sheriff, J., Claiborne, T. E., Slepian, M. J., Redaelli, A., and Bluestein, D., 2015, "Hemodynamic and Thrombogenic Analysis of a Trileaflet Polymeric Valve Using a Fluid-Structure Interaction Approach," J. Biomech., 48(13), pp. 3650-3658.

[32] Lau, K. D., Diaz, V., Scambler, P., and Burriesci, G., 2010, "Mitral Valve Dynamics in Structural and Fluid-Structure Interaction Models," Med. Eng. Phys., 32(9), pp. 1057-1064.

[33] Weinberg, E. J., and Kaazempur Mofrad, M. R., 2007, "Transient, ThreeDimensional, Multiscale Simulations of the Human Aortic Valve," Cardiovasc. Eng., 7(4), pp. 140-155.

[34] Einstein, D. R., Kunzelman, K. S., Reinhall, P. G., Nicosia, M. A., and Cochran, R. P., 2005, "Non-Linear Fluid-Coupled Computational Model of the Mitra Valve," J. Heart Valve Dis., 14(3), pp. 376-385.

[35] Hallquist, J. O., 2006, "Simplified Arbitrary Lagrangian-Eulerian," LS-DYNA Theory Manual, Livermore Software Technology Corporation (LSTC), Livermore, CA, Chap. 14.

[36] Marom, G., 2015, "Numerical Methods for Fluid-Structure Interaction Models of Aortic Valves," Arch. Comput. Methods Eng., 22(4), pp. 595-620.

[37] Hallquist, J. O., 2007, LS-DYNA Keyword User's Manual, Livermore Software Technology Corporation (LSTC), Livermore, CA

[38] Wu, J., Liu, J., and Du, Y., 2007, "Experimental and Numerical Study on the Flight and Penetration Properties of Explosively-Formed Projectile," Int. J. Impact Eng., 34(7), pp. 1147-1162.

[39] Daily, B. B., Pettitt, T. W., Sutera, S. P and Pierce, W. S., 1996, "PierceDonachy Pediatric VAD: Progress in Development," Ann. Thorac. Surg., 61(1), pp. 437-443

[40] Bluestein, D., Chandran, K. B., and Manning, K. B., 2010, "Towards NonThrombogenic Performance of Blood Recirculating Devices," Ann. Biomed. Eng., 38(3), pp. 1236-1256.

[41] Liu, Q., Runt, J., Felder, G., Rosenberg, G., Snyder, A. J., Weiss, W. J., Lewis J., and Werley, T., 2000, "In Vivo and In Vitro Stability of Modified Poly(urethaneurea) Blood Sacs," J. Biomater. Appl., 14(4), pp. 349-366.

[42] Bachmann, C., Hugo, G., Rosenberg, G., Deutsch, S., Fontaine, A., and Tarbell, J. M., 2000, "Fluid Dynamics of a Pediatric Ventricular Assist Device," Artif Organs, 24(5), pp. 362-372.

[43] Baldwin, J. T., Borovetz, H. S., Duncan, B. W., Gartner, M. J., Jarvik, R. K. Weiss, W. J., and Hoke, T. R., 2006, "The National Heart, Lung, and Blood Institute Pediatric Circulatory Support Program," Circulation, 113(1), pp. $147-155$.

[44] Fraser, K. H., Taskin, M. E., Griffith, B. P., and Wu, Z. J., 2011, "The Use of Computational Fluid Dynamics in the Development of Ventricular Assist Devices," Med. Eng. Phys., 33(3), pp. 263-280.

[45] Konig, C. S., Clark, C., and Mokhtarzadeh-Dehghan, M. R. 1999 "Investigation of Unsteady Flow in a Model of a Ventricular Assist Device by Numerical Modelling and Comparison With Experiment," Med. Eng. Phys. 21(1), pp. 53-64.

[46] Behbahani, M., Behr, M., Hormes, M., Steinseifer, U., Arora, D., Coronado, O., and Pasquali, M., 2009, "A Review of Computational Fluid Dynamics Analysis of Blood Pumps,” Eur. J. Appl. Math., 20(4), pp. 363-397.

[47] Medvitz, R. B., Reddy, V., Deutsch, S., Manning, K. B., and Paterson, E. G., 2009, "Validation of a CFD Methodology for Positive Displacement LVAD Analysis Using PIV Data," ASME J. Biomech. Eng., 131(11), p. 111009 . 